\title{
Neutropenia, Inflammation, and the Kinetics of Transfused Neutrophils in Rabbits
}

\author{
Marc S. Rosenshein, Thomas H. Price, and David C. Dale, Puget Sound \\ Blood Center, Seattle Washington 98104; Division of Hematology, \\ Department of Medicine, University of Washington, Seattle, \\ Washington 98195
}

A B S T RACT A rabbit model was used to study the effects of neutropenia and inflammation on the intravascular distribution, survival, and tissue accumulation of transfused neutrophils. Donor blood labeled with $\left[{ }^{3} \mathrm{H}\right]$ thymidine was infused into normal or neutropenic (vinblastine treated) animals. Inflammation was created by subcutaneous implantation of polyvinyl sponges, some with added endotoxin. Initial circulating neutrophil pool recovery, survival, and inflammatory site accumulation of labeled neutrophils were measured.

Neutropenia was associated with a relative increase in the marginal pool size, manifested by a diminished initial circulating pool (CNP) recovery of transfused cells. The CNP recovery was directly proportional to recipient neutrophil count. Neutropenia had no effect on the intravascular survival of transfused cells and was accompanied by only a modest decrease in the inflammatory site recovery of the transfused neutrophils ( $10.4 \pm 5.4$ vs. $14.4 \pm 4.0 \%$ in normals).

Inflammation in the form of subcutaneous polyvinyl sponges was accompanied by an increase in margination with initial CNP recoveries of $24.3 \pm 4.7$ and $27.6 \pm 8.8 \%$ at zero and $4 \mathrm{~h}$ after implantation respectively (normal, $38.2 \pm 9.9 \%$ ). Transit through the CNP was hastened by inflammation with a $t_{1 / 2}$ of $2.02 \pm 0.72 \mathrm{~h}$ (normal, $3.2 \pm 1.0 \mathrm{~h}$ ).

Addition of endotoxin to the sponges further perturbed cell kinetics. CNP recoveries were considerably lower and half-lifes were initially shorter and subsequently uninterpretable in studies done after endotoxin sponge insertion. Inflammatory site accumulation was markedly diminished to $7.4 \pm 1.9 \%$ of injected neutrophil label in the endotoxin sponge animals, sug-

This work was presented in part at the 70th Annual Meeting of the American Society for Clinical Investigation, San Francisco, Calif., 1 May 1978, and published in abstract form. 1978. Clin. Res. 26: 507A.

Received for publication 8 February 1979 and in revised form 9 April 1979. gesting that many of the transfused cells were functionally unavailable rather than marginated. These studies demonstrate that neutropenia and inflammation with or without endotoxin markedly alter the kinetics of transfused neutrophils and that CNP recovery of transfused cells is not necessarily predictive of their inflammatory site accumulation.

\section{INTRODUCTION}

Blood neutrophils are distributed between two freely communicating compartments, the circulating and marginal neutrophil pools. (1). It is generally accepted that neutrophils enroute to extravascular inflammatory sites leave the circulation directly from the marginal pool. Because of its accessibility, the circulating neutrophil pool $(\mathrm{CNP})^{1}$ has been extensively studied, and the normal kinetics of cells in this compartment are relatively well defined. The marginal pool, on the other hand, has only been evaluable by indirect $(1,2)$ or nonquantitative morphologic (3) methods, and information on the behavior of cells in this compartment is largely speculative. How the intravascular localization of neutrophils within these compartments relates quantitatively to the neutrophils' ability to reach inflammatory sites is virtually unknown.

Because of the development of neutrophil transfusion, it has become increasingly important to understand the factors controlling the intravascular distribution of neutrophils and their kinetics. In general, the assumptions used to evaluate neutrophil transfusions have been based on kinetic studies performed on normal volunteers $(1,2)$. However, it is well established from patient studies (4-6) that posttransfusion neutrophil count increments are far less than those predicted by kinetic studies in normal subjects. Early studies of neutrophil transfusion also demonstrated

${ }^{1}$ Abbreviation used in this paper: CNP, circulating neutrophil pool. 
that the blood recovery of transfused neutrophils is related to the recipient's pretransfusion neutrophil count (7). That this may be due in part to a relative increase in marginal pool size is suggested by studies showing larger than predicted neutrophil count increments in neutropenic subjects infused with epinephrine (8). There are also marked alterations in blood neutrophil kinetics associated with both acute (9) and chronic $(10,11)$ infections. These observations suggest that predictions derived from studies on normal subjects are not applicable to the usual transfusion recipient, and that recipient variables, such as neutrophil count and the presence or absence of infection should be considered in evaluating the kinetics of transfused neutrophils. Using a rabbit model developed in our laboratory we have attempted to investigate the effects of neutropenia, inflammation, and endotoxin on the intravascular distribution, blood kinetics, and the inflammatory site accumulation of transfused neutrophils.

\section{METHODS}

New Zealand white rabbits of either sex, weighing $\cong 3 \mathrm{~kg}$, were used for all experiments. Recipient animals were used only once to avoid sensitization.

Blood containing labeled neutrophils was obtained by infusing donor animals with $2.0 \mathrm{mCi}\left[{ }^{3} \mathrm{H}\right]$ thymidine and harvesting $50-80 \mathrm{ml}$ of blood by cardiac puncture $72 \mathrm{~h}$ later, at the time of maximum neutrophil labeling. Sterile acid citrate dextrose solution, formula $\mathrm{A}$, was used as the anticoagulant for this and all other blood specimens. $20 \mathrm{ml}$ of this blood was saved as a standard and neutrophil specific activity determined in triplicate using the following procedures.

Leukocyte counts and 200 cell differential counts were performed on all specimens. Neutrophils were isolated by centrifugation on Ficoll-Hypaque (Ficoll, Sigma Chemical Co., St. Louis, Mo.; Hypaque, Winthrop Laboratories, New York) (1.077-1.078 sp gr) followed by dextran (200,000$300,000 \mathrm{~mol} \mathrm{wt})$ sedimentation of the erythrocyte granulocyte layer at $1 \mathrm{~g}$, and serial $0.87 \% \mathrm{NH}_{4} \mathrm{Cl}$ washes to lyse the remaining erythrocytes. After determining the cell count of the final neutrophil suspension, a known volume was centrifuged at $2,000 \mathrm{~g}$ and the resulting pellet was dissolved in $1 \mathrm{ml}$ NCS (NCS tissue solubilizer, Amersham Corp., Arlington Heights, Ill.). $16 \mathrm{ml}$ of scintillation fluid were added and the radioactivity was counted in a liquid scintillation counter (Packard Instrument Co., Inc., Downers Grove, Ill.). Neutrophil specific activity of the standard was calculated and expressed as counts per minute $/ 10^{6}$ cells. The final cell pellets were $99 \%$ granulocytes. Neutrophil yield from the initial specimens was consistently $35-50 \%$.

Blood neutrophil kinetics were measured after infusion of $10 \mathrm{ml}$ of labeled donor blood to recipients via the marginal ear vein. Serial 6-ml blood specimens were obtained via cardiac puncture $10 \mathrm{~min}$ after the midpoint of the infusion and also at 60, 150, 240,300, and $360 \mathrm{~min}$. Leukocyte counts and differential smears were performed on all specimens, neutrophils were isolated and activity determined as described above. Results were expressed as the percentage of injected neutrophil label recovered in the CNP using the formula: CNP recovery $=$ (counts per $\min$ per milliliter $\times$ blood volume/counts per minute injected) $\times 100 \%$, with counts per minute injected $=$ specific activity of the standard $\times$ (neutrophil) standard $\times 10 \mathrm{ml}$. Blood volume was considered to be $50 \mathrm{ml} / \mathrm{kg}$. The recovery data points were plotted against time on semilogarithmic paper, and neutrophil survival curves determined using the method of least squares. Initial CNP recovery was the point where the curve crossed the ordinate (zero time). Blood neutrophil half-life was determined from the slope of the survival curve. To pool data from similar groups of animals, values obtained at the same time points were used and composite survival curves were constructed using the mean recovery values for each time point. Statistical significance was determined between various groups using the Student's two-tailed $t$ test.

Inflammatory sites were created in some recipient animals by insertion of subcutaneous polyvinyl sponges. Polyvinyl sponges packed in formalin (Unipoint Industries Inc., High Point, N. C.) were cut into $3 \times 3 \times 1 / 3-\mathrm{cm}$ pieces and washed multiple times in tap water. They were sterilized by boiling for $10 \mathrm{~min}$ in deionized water and rinsed in sterile normal saline solution before use. Rabbits were tranquilized with $3 \mathrm{mg}$ acepromazine and anesthetized with pentobarbital. The skin of the back was shaved and washed and six sponges inserted into the subcutaneous fascial space via a 3- to 4-cm midline incision after creation of a subcutaneous pocket by blunt dissection. The sponges were inserted lying flat, at least $1-\mathrm{cm}$ lateral to the skin incision. The incision was closed with metal clips and sealed with flexible collodion. $10 \mathrm{ml}$ of labeled blood was infused $4 \mathrm{~h}$ after sponge insertion. At $24 \mathrm{~h}$, the animals were sacrificed and the sponges removed. After air drying, the sponges were compressed into pellets and burned in a tissue oxidizer (Tri-Carb model 305, Packard Instrument Co., Inc.). Activity was counted in a liquid scintillation counter using Oxyfluor (New England Nuclear, Boston, Mass.) as the scintillant. The accumulation of radioactivity in the sponges was expressed as a percentage of the injected neutrophil label. As demonstrated by this laboratory (12), donor blood components were labeled to various degrees after injecting $\left[{ }^{3} \mathrm{H}\right]$ thymidine. However, except for an apparently constant contribution from labeled plasma equivalent to $\cong 1.5 \%$ of neutrophil counts per minute injected, essentially all the label accumulating in the sponges is neutrophil associated.

In one group of animals, to assess the effects of an inflammatory focus on blood kinetics, labeled blood was infused either immediately or 3-4 $\mathrm{h}$ after sponge insertion and blood kinetics were studied over the subsequent $6 \mathrm{~h}$ as detailed above. Sponges were removed and processed at $24 \mathrm{~h}$.

In another group of animals, $1 \mathrm{mg}$ of Salmonella typhosa endotoxin (Difco Laboratories, Detroit, Mich.) dissolved in $0.2 \mathrm{ml}$ saline was added to each sponge before insertion. Blood neutrophil kinetic and sponge accumulation experiments were then carried out as described above either immediately or 3-4 h after sponge insertion. Limulus amebacyte gelation assays for endotoxin (13) were kindly performed by Dr. Ron Elin, NIH, Bethesda, Md., on plasma specimens from several of these animals at various times after endotoxin sponge insertion.

A final group of animals was made neutropenic with intravenous vinblastine, $0.75 \mathrm{mg} / \mathrm{kg}$, given $48-72 \mathrm{~h}$ before either blood kinetic or sponge accumulation experiments. Serial neutrophil counts were performed on these animals. Techniques and calculations for these experiments in neutropenic animals were identical to those described for normal animals. Blood kinetic and sponge accumulation experiments were also initiated at various times after the injection of vinblastine to assess the occurrence and duration of direct drug-related effects on the neutrophil kinetics. Total body irradiation with $1,200 \mathrm{R}$ was also attempted to induce neutropenia but was unacceptable because of a very high mortality rate in the first $24 \mathrm{~h}$ after irradiation. Nitrogen mustard was also tried and 


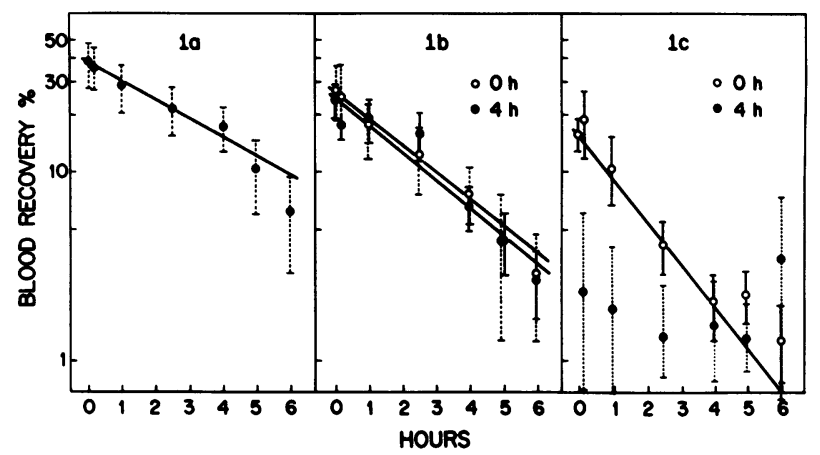

Figure 1 Disappearance of labeled neutrophils from blood after infusion of $\left[{ }^{3} \mathrm{H}\right]$ thymidine-labeled neutrophils. Vertical bars represent \pm 1 SD $(a)$ Normal recipient animals $(n=10)$. (b) Recipient animals with cells injected immediately $(n=16)$ or $4 \mathrm{~h}(n=8)$ after polyvinyl sponge insertion. (c) Recipient animals with cells injected immediately $(n=5)$ or $4 \mathrm{~h}(n=7)$ after endotoxin sponge insertion.

rejected because it routinely caused inflammation and swelling along the course of the vein used for infusion. Cyclophosphamide in single doses adquate to cause severe neutropenia was accompanied by unacceptable early mortality as well as gross hematuria in the majority of animals.

\section{RESULTS}

Studies of animals with normal neutrophil counts. Blood neutrophil kinetics were evaluated in a group of 10 normal rabbits. A composite neutrophil survival curve for these animals is shown in Fig. 1a. Extrapolated mean initial recovery of injected labeled neutrophils in the $\mathrm{CNP} \pm 1 \mathrm{SD}^{2}$ was $38.2 \pm 9.9 \%$. There was a subsequent exponential disappearance of labeled cells from the circulation with a $t_{1 / 2}$ of $3.2 \pm 1.0 \mathrm{~h}$. Sponge accumulation studies on 20 normal rabbits revealed that $14.4 \pm 4.0 \%$ of injected granulocyte label was recovered in the six sponges $24 \mathrm{~h}$ after insertion.

The effects of a localized inflammatory focus on blood neutrophil kinetics were studied in 16 rabbits immediately after sponge insertion (zero $h$ ) and in 8 rabbits beginning $4 \mathrm{~h}$ after sponge insertion $(4 \mathrm{~h}$ ) (Table I). The composite survival curves for these groups are shown in Fig. 1b. In the "zero-h" group, the initial blood recovery was $27.6 \pm 8.8 \%$, compared to the normal blood recovery of $38.2 \pm 9.9 \%$ In the " $4 \mathrm{~h}$ " group, the blood recovery was $24.3 \pm 4.7 \%$. The initial blood recovery in both the zero- and 4-h groups was significantly less than in the normal animals $(P<0.05)$. Half-life was significantly shortened in sponge bearing animals to $2.02 \pm 0.72 \mathrm{~h}(P<0.01)$.

In another group of experiments, addition of endotoxin to the sponges before insertion was found to further perturb blood neutrophil kinetics, as well as

${ }^{2}$ All subsequent data are presented as arithmetic means \pm 1 SD unless otherwise indicated.
TABLE I

Effects of Inflammation and Endotoxin on Neutrophil Kinetics

\begin{tabular}{lccc}
\hline & $\begin{array}{c}\text { Initial CNP } \\
\text { recovery }\end{array}$ & \multicolumn{1}{c}{$\mathrm{t}_{\mathrm{y} / 2}$} & $\begin{array}{c}\text { Sponge } \\
\text { accumulation }\end{array}$ \\
\hline & $\%$ & $h$ & $\%$ \\
Normal & $38.2 \pm 9.9$ & $3.2 \pm 1.00$ & \\
Cells infused at 0 h & & & \\
$\quad$ Sponge only & $27.6 \pm 8.8$ & $2.02 \pm 0.72$ & $13.9 \pm 2.7^{*}$ \\
$\quad$ Endotoxin sponge & $16.0 \pm 3.7$ & $1.48 \pm 0.22$ & $5.7 \pm 2.0^{*}$ \\
Cells infused at 4 h & & & \\
$\quad$ Sponge only & $24.3 \pm 4.7$ & $2.02 \pm 0.72$ & $14.4 \pm 4.0$ \\
Endotoxin sponge & $2.4 \pm 2.0$ & $\mathrm{NS}$ & $7.4 \pm 1.9$ \\
\hline
\end{tabular}

* Sponge accumulation data from zero-h infusions not listed in text.

inflammatory site accumulation (Table I; Fig. 1c). In five normal animals studied immediately after endotoxinsponge implantation, the mean blood recovery was 16.0 $\pm 3.7 \%$ and the $t_{1 / 2}$ was $1.48 \pm 0.22 \mathrm{~h}$. When the experiments were initiated $4 \mathrm{~h}$ after insertion, the blood recovery was only $2.4 \%$ and no half-life could be calculated since interpretable survival curves could not be constructed from subsequent data points. Although previous studies from this laboratory have shown that endotoxin increases the overall number of cells accumulating in the subcutaneous sponges in normal animals (12), the percentage of labeled transfused cells accumulating was decreased to $7.4 \pm 1.9 \%$ (Fig. 5) in the endotoxin sponge-bearing animals $(P<0.05)$. No endotoxemia was detectable using the Limulus amebacyte gelation assay in three animals tested 15 min, 4 and $24 \mathrm{~h}$ after endotoxin sponge insertion.

Studies of neutropenic animals. The change in neutrophil counts after administration of $0.75 \mathrm{mg} / \mathrm{kg}$ of vinblastine to four rabbits is shown in Fig. 2. The

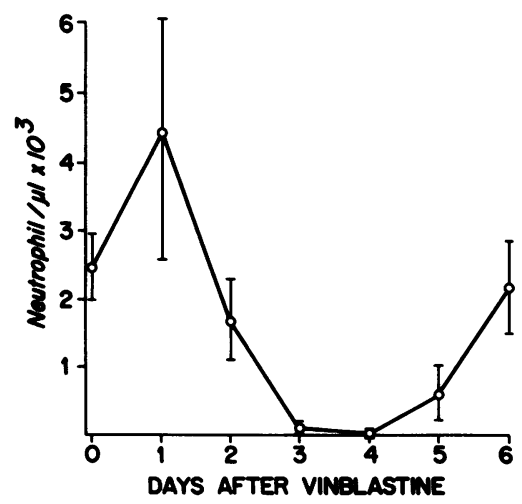

Figure 2 Neutrophil counts after vinblastine $0.75 \mathrm{mg} / \mathrm{kg}$ in four rabbits (mean $\pm 1 \mathrm{SD}$ ). 
animals were uniformly severely neutropenic on days 3 and 4 after vinblastine administration. Other than occasional animals who developed diarrhea, no appreciable complications of vinblastine administration were noted.

To investigate the relationship between the recipient neutrophil count and the fate of transfused cells, blood kinetics were studied in 26 animals with a wide range of neutrophil counts. Some had received no vinblastine, and others had received vinblastine $48-72 \mathrm{~h}$ before beginning the kinetic studies. There was a direct relationship between recipient neutrophil count and the initial CNP recovery of transfused cells (Fig. 3). This relationship was evident throughout the neutropenic and normal ranges, varying from initial CNP recoveries of $<1 \%$ in animals with neutrophil counts under $50 / \mu l$ to recoveries of $\cong 50 \%$ in animals with neutrophil counts of $4,000-5,000 / \mu l$.

In contrast, half-life was not related to neutrophil count. Fig. 4 shows half-life plotted against recipient neutrophil count. There was no significant difference in half-life between severely neutropenic and normal animals. Thus, although a smaller fraction of cells are in the CNP initially in the neutropenic, uninflamed animal, those remaining leave the circulation with a normal half-life.

In a group of 16 neutropenic animals, all with neutrophil counts of $\langle 250 / \mu \mathrm{l}$, mean sponge accumulation at $24 \mathrm{~h}$ was $10.4 \pm 5.4 \%$ as compared to a normal of $14.4 \%$ (Fig. 5). Although this difference was statistically significant $(P<0.05)$ the decrease in inflammatory site accumulation was only $28 \%$ despite a 10 -fold decrease in mean CNP recovery in animals with similar degrees of neutropenia from 38.2 to $3.8 \%$.

When labeled cells were infused immediately after an intravenous injection of vinblastine, initial CNP recovery was quite low suggesting that the vinblastine itself acutely alters neutrophil kinetics. However, at 24 and $48 \mathrm{~h}$ after vinblastine, blood kinetics returned to a pattern comparable to normal animals (Table II). Sponge accumulation of radioactivity was also markedly diminished in animals given vinblastine immediately before infusing labeled blood, but these values also increased towards normal values at 24 and $48 \mathrm{~h}$ after vinblastine. Thus, although vinblastine did have

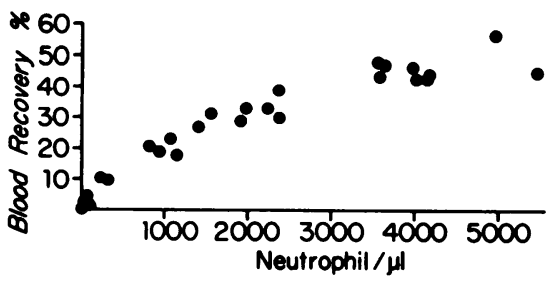

FIGURE 3 Relationship of initial CNP recovery of labeled cells to recipient neutrophil count.

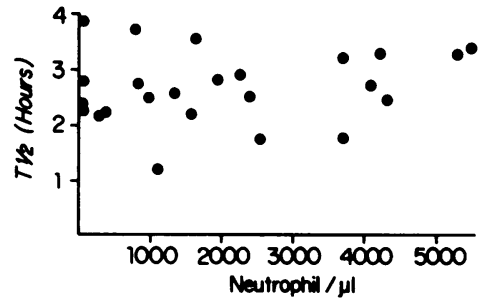

FIgURE 4 Relationship of neutrophil blood half-life to recipient neutrophil count.

direct acute effects on neutrophil blood kinetics and inflammatory site accumulation in the first $24 \mathrm{~h}$ after administration, these effects appeared to resolve before the animals became neutropenic.

When endotoxin was added to the sponges in neutropenic animals, blood kinetics could not be evaluated because of low initial recoveries $(<1 \%)$, and uninterpretable survival curves. This group of 11 animals had the lowest sponge accumulation of any group, $2.4 \pm 0.5 \%$ of the injected granulocyte label (Fig. 5).

\section{DISCUSSION}

The importance of recipient characteristics in determining the kinetics and fate of transfused neutrophils has been suggested by several investigators. In their early work on leukocyte transfusion, Morse et al. (7) suggested that the recovery and survival of transfused neutrophils depended on both the recipient's initial neutrophil count and the presence or absence of fever. Joyce et al. (8) studied the marginal neutrophil pool size in normal and neutropenic subjects, and demonstrated that the postepinephrine neutrophil increment was inversely proportional to the base-line neutrophil count. Studies of neutrophil kinetics in

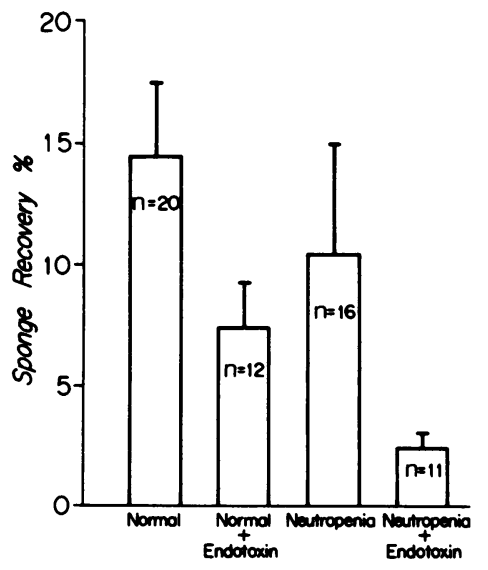

Figure 5 Percentage of transfused labeled neutrophils accumulating in subcutaneous polyvinyl sponges over $24 \mathrm{~h}$. Values shown are means \pm 1 SD for the various groups of animals. 
TABLE II

Effects of Vinblastine on Neutrophil Kinetics

\begin{tabular}{cccc}
\hline & $\begin{array}{c}\text { Initial CNP } \\
\text { recovery }\end{array}$ & $\mathrm{t}_{1 / 2}$ & $\begin{array}{c}\text { Sponge } \\
\text { accumulation }\end{array}$ \\
\hline $\begin{array}{c}\text { Normal sponge- } \\
\text { bearing rabbit }\end{array}$ & $24.3 \pm 4.7$ & $2.02 \pm 0.72$ & $14.4 \pm 4.0$ \\
$\begin{array}{c}0 \text { h after Velban } \\
(n=3)\end{array}$ & $4.2 \pm 1.8$ & $2.3 \pm 0.3$ & $4.1 \pm 2.4$ \\
$\begin{array}{c}24 \mathrm{~h} \text { after Velban } \\
(n=3)\end{array}$ & $33.2 \pm 2.5$ & $2.9 \pm 0.7$ & $10.6 \pm 2.7$ \\
$\begin{array}{c}48 \mathrm{~h} \text { after Velban } \\
(n=3)\end{array}$ & $26.4 \pm 8.5$ & $2.5 \pm 1.0$ & $11.2 \pm 1.7$ \\
\hline
\end{tabular}

acute (9) and chronic $(10,11)$ infection have shown increased margination and a shortened half-life in early acute infection, followed by an expansion of the total blood neutrophil pool and normalization or lengthening of half-life in established or chronic infection.

The animal studies reported here demonstrate that abnormalities of the transfusion recipient may have profound effects on the blood kinetics and inflammatory site accumulation of transfused neutrophils. These effects can confidently be attributed to the recipient variables since the labeled cells were administered as freshly collected whole blood, eliminating collection or storage induced artifacts in cell function as a variable.

The initial CNP recovery of transfused neutrophils was directly related to the recipients' neutrophil count. This suggests an alteration of the normal equilibrium between the CNP and marginal pool in the neutropenic recipient animals, with localization of a large percentage of the transfused cells in the marginal pool at the expense of the circulating pool. In the neutropenic animals, inflammatory site accumulation was close to normal. This suggests that the majority of cells, though not demonstrable in the CNP, were nonetheless functionally available in the marginal pool and able to enter the inflammatory exudate.

Inflammation in the form of subcutaneously implanted polyvinyl sponges affected both intravascular distribution and survival of the transfused cells. Both CNP recovery and the intravascular survival of transfused cells were diminished in sponge-bearing animals as compared to normals, suggesting that both increased margination and an increased demand for neutrophils were created by the presence of the inflammatory site. All survival data were expressed in terms of radioactivity per volume of blood, rather than as neutrophil specific activity, eliminating the possibility that the shortened half-life was a result of the influx of unlabeled neutrophils from the marrow.
Endotoxin has been shown to have a variety of effects on neutrophils and their kinetics. Many of these effects are similar to those induced by bacterial infection and phagocytosis (15). In both animals and man, small intravenous doses of endotoxin induce a transient neutropenia followed by release of marrow neutrophils into the blood $(2,16)$. It has been shown that endotoxin increases the adherence of granulocytes to nonbiologic surfaces (17). Other studies $(18,19)$ have demonstrated that fewer neutrophils are able to migrate to tissue sites early after endotoxin administration during the period of neutropenia.

These studies in animals with endotoxin sponges may help to explain some of the difficulties encountered in assessing the results of neutrophil transfusion in the infected patient. Early after implantation the CNP recovery and survival results were qualitatively similar to those seen in sponge-bearing animals without endotoxin, although the CNP recovery was lower and the half-life values shorter (Fig. $1 b$ and $c$ ). This suggests that the endotoxin sponge was a more potent inflammatory stimulus than the sponge alone. However, when the blood kinetic study was begun later, very few cells could be recovered in the CNP, and no meaningful survival curves or half-lives could be derived. Although the low initial CNP recovery in these endotoxin sponge animals was similar to that seen in neutropenic animals without implanted sponges, the subsequent kinetics were quite different. In neutropenic animals, despite the low CNP recovery, the half-life of the cells in circulation was normal, while in the endotoxin animals the half-life was initially shortened and later unmeasureable. This suggets that the nonrecovered neutrophils in the endotoxin sponge animals were sequestered or lost and not in dynamic equilibrium with the circulating pool.

Inflammatory site accumulation of the transfused cells was also significantly altered by endotoxin. Although the addition of endotoxin to the sponges increases the total number of cells arriving at the inflammatory site (12), the work presented here shows that endotoxin decreases the proportion of labeled transfused cells accumulating in the inflammatory focus. This apparent paradox can be explained by one of two mechanisms. It is possible that the transfused labeled cells are infused at the time of maximal endotoxin effect and may therefore be damaged and unable to accumulate at the inflammatory site, whereas the larger number of unlabeled endogenous cells are released from the marrow later, remain functionally intact, and could account for most of the cells in the sponges. Alternatively, the endotoxin may induce an increase in overall neutrophil turnover by creating sites of neutrophil loss or sequestration that compete with the sponges for both labeled and unlabeled neutrophils, 
resulting in the smaller percentage of transfused cells in the sponges. Whichever mechanism is operative, a significantly smaller fraction of the transfused cells localize at an inflammatory site in the endotoxin-treated animals, regardless of their neutrophil counts. The inflammatory site accumulation in neutropenia was $72 \%$ of that in normal animals while in the endotoxin sponge experiments it varied from 14 to $50 \%$ depending on the animals' neutrophil count. These data support sequestration or loss of neutrophils in the endotoxin model, as opposed to their localization in a functional marginal pool. The animals that were both neutropenic and bearing endotoxin sponges showed the lowest inflammatory site accumulation of transfused neutrophils of any group. Interestingly, this situation perhaps most closely approximates the usual clinical circumstances for which neutrophil transfusion is considered, the neutropenic patient with significant systemic infection.

\section{ACKNOWLEDGMENTS}

We gratefully acknowledge the technical assistance of Stan Corpuz and the secretarial assistance of Bette Whitney.

This work was supported by the Blood Research and Demonstration Center grant HL-17625 and by National Institutes of Health grant HL-06242.

\section{REFERENCES}

1. Athens, J., S. Raab, O. Haab, A. Mauer, H. Ashenbrucker, G. Cartwright, and M. Wintrobe. 1961. Leukokinetic studies. III. The distribution of granulocytes in the blood of normal subjects. J. Clin. Invest. 40: 159-164.

2. Athens, J., O. Haab, S. Raab, A. Mauer, H. Ashenbrucker, G. Cartwright, and M. Wintrobe. 1961. Leukokinetic studies. IV. The total blood, circulating and marginal granulocyte pools and the granulocyte turnover rate in normal subjects. J. Clin. Invest. 40: 989-995.

3. Vejlens, G. 1938. The distribution of leukocytes in the vascular system. Acta Pathol. Microbiol. Scand. 15 (Suppl. 13): 1-239.

4. McCredie, K., J. Hester, and E. Freireich. 1977. Leukocyte collection and transfusion physiology. Exp. Hematol. (Copenhagen). 5(Suppl. 1): 33-38.

5. Herzig, R., G. Herzig, R. Graw, Jr., M. Bull, and K. Ray. 1977. Successful granulocyte transfusion therapy for gramnegative septicmia. N. Engl. J. Med. 296: 701-705.

6. Alavi, J., R. Root, I. Djerassi, A. Evans, S. Gluckman,
R. MacGregor, D. Guerry, A. Schreiber, J. Shaw, P. Koch, and R. Cooper. 1977. A randomized clinical trial of granulocyte transfusions for infection in acute leukemia. N. Engl. J. Med. 296: 706-711.

7. Morse, E., E. Freireich, P. Carbone, W. Bronson, and E. Frei, III. 1966. The transfusion of leukocytes from donors with chronic myelocytic leukemia to patients with leukopenia. Transfusion (Phila.). 6: 183-192.

8. Joyce, R., D. Boggs, U. Hasiba, and C. Srodes. 1976. Marginal neutrophil pool size in normal subjects and neutropenic patients as measured by epinephrine infusion. J. Lab. Clin. Med. 88: 614-620.

9. Marsh, J., D. Boggs, G. Cartwright, and M. Wintrobe. 1967. Neutrophil kinetics in acute infection. J. Clin. Invest. 46: 1943-1953.

10. Athens, J., O. Haab, S. Raab, D. Boggs, H. Ashenbrucker, G. Cartwright, and M. Wintrobe. 1965. Leukokinetic studies. XI. Blood granulocyte kinetics in polycythemia vera, infection, and myelofibrosis. J. Clin. Invest. 44: 778-788.

11. Galbraith, P., L. Valberg, and M. Brown. 1965. Patterns of granulocyte kinetics in health, infection and in carcinoma. Blood. 25: 683-692.

12. Price, T., D. Dale. 1977. Neutrophil preservation. The effect of short term storage on in vivo kinetics. J. Clin. Invest. 59: 475-480.

13. Elin, R., and S. Wolff. 1973. Nonspecificity of the Limulus amebocyte Lysate test: positive reactions with polynucleotides and proteins. J. Infect. Dis. 128: 349-352.

14. Deubelbeiss, V., J. Dancey, L. Harker, and C. Finch. 1975. Neutrophil kinetics in the dog. J. Clin. Invest. 55: 833-839.

15. Cline, M., K. Melmon, W. Davis, and H. Williams. 1968. Mechanism of endotoxin interaction with human leukocytes. Br. J. Haematol. 15: 539-547.

16. Berthrong, M., and L. Cluff. 1953. Studies of the effect of bacterial endotoxins on rabbit leukocytes. I. Effect of intravenous injection of the substances with and without induction of the local Shwartzman reaction. J. Exp. Med. 98: 331-347.

17. MacGregor, R. 1977. Granulocyte adherence changes induced by hemodialysis, endotoxin, epinephrine, and glucocorticoids. Ann. Intern. Med. 86: 35-39.

18. Fekety, F. 1969. Skin window studies of the effect of endotoxin upon the acute inflammatory response. John Hopkins Med. J. 124: 291-295.

19. Mulholland, J., and L. Cluff. 1964. The effect of endotoxin upon susceptibility to infection: the role of the ganulocyte, in M. Sandy and W. Braun, editors. Bacterial Endotoxins: Proceedings, Symposium on Bacterial Endotoxins. New Brunswick Institute of Microbiology of Rutgers University. 211. 